\title{
Quantitative Evaluation of Turbulence Compensation
}

\author{
Adam W.M. van Eekeren", Klamer Schutte, Judith Dijk, \\ Piet B.W. Schwering \\ TNO, PO Box 96864, 2509 JG The Hague, The Netherlands
}

\begin{abstract}
A well-known phenomena that diminishes the recognition range in infrared imagery is atmospheric turbulence. In literature many methods are described that try to compensate for the distortions caused by atmospheric turbulence. Most of these methods use a global processing approach in which they assume a global shift and a uniform blurring in all frames. Because the effects of atmospheric turbulence are often spatial and temporal varying, we presented previous year a turbulence compensation method that performs local processing leading to excellent results. In this paper an improvement of this method is presented which uses a temporal moving reference frame in order to be capable of processing imagery containing moving objects as well as blur estimation to obtain adaptive deconvolution. Furthermore our method is evaluated in a quantitative way, which will give a good insight in which components of our method contribute to the obtained visual improvements.
\end{abstract}

Keywords: Turbulence compensation, deconvolution, image restoration, super-resolution, quantitative evaluation

\section{INTRODUCTION}

Atmospheric turbulence is a well-known phenomenon which deteriorates imagery, captured over long ground-to-ground ranges. This diminishes the recognition range and it is therefore important to compensate visual degradation due to atmospheric turbulence. Turbulence compensation is a challenging problem because 1) the amount of turbulence is spatially varying due to anisoplanatic conditions while the isoplanatic angle, i.e. the angle over which the turbulence can be assumed constant, varies with atmospheric conditions and 2) the amount of turbulence varies significantly in time.

According to Roggeman and Welsh [1] there are three different approaches to compensate for turbulence effects in images: 1) adaptive optics techniques, 2) post-processing techniques and 3) a combination of both techniques. An overview of different turbulence compensation methods can be found in $[2,3]$.

In this paper we will focus on a post-processing technique that is also capable of processing imagery containing slow moving objects. This method is an improvement of earlier published work [4,5]. The improvement consists basically of two parts: 1) using a temporal moving reference frame, which enables the processing of slow moving objects and 2) using blur estimation, which improves the deconvolution part of our method.

In literature many software post-processing techniques are described, such as the methods described in [6,7] which perform first a global registration followed by deblurring. There also exist methods that perform local registration to cope with anisoplanatic conditions $[8,9]$. Comparable to [9], we developed a post-processing technique based on multiframe super-resolution reconstruction. Such an approach incorporates registration, temporal image fusion and deblurring. Our proposed method copes with anisoplanatic conditions by using local registration. The major differences with the approach of [9] are that we perform frame selection, blur estimation and that our registration is less computational complex.

For target recognition purposes it is important to evaluate the image quality improvement of turbulence compensation methods. This task can be done by various methods. Some methods need specific targets in the scene, such as the TOD method [10] and MRTD method [11]. Other methods can be calculated on any scene, such as the Structural SImilarity Measure (SSIM) [12] and the Video Quality Measure (VQM) [13]. An overview of various evaluation techniques are described by Dijk et al.[14]. In this paper we measure the blur and the tilt in the original and processed imagery to obtain

*adam.vaneekeren@tno.nl; phone +31 8886 64047; www.tno.nl

Infrared Imaging Systems: Design, Analysis, Modeling, and Testing XXIV, edited by

Gerald C. Holst, Keith A. Krapels, Proc. of SPIE Vol. 8706, 87060K · C 2013 SPIE

CCC code: $0277-786 X / 13 / \$ 18 \cdot$ doi: $10.1117 / 12.2015371$ 
quantitative image quality measures. These general, basic measures give a good indication of the image quality improvement of our proposed method.

The setup of the paper is as follows. In Section 2 the assumed imaging model and the proposed turbulence compensation method are described. In Section 3 the quantitative quality measures are described that are used to evaluate the results of the proposed method. The evaluation results are presented in Section 4 and finally conclusions will be drawn in Section 5 .

\section{TURBULENCE COMPENSATION METHOD}

\subsection{Imaging model}

This section describes the assumed imaging model. The camera's field-of-view, the scene, is modeled as a properly sampled 2D HR image $\mathbf{Z}$ (see Figure 1). It is assumed that this HR image is free of degradation to motion, blur and noise. The output frames $\mathbf{y}_{k}$ are a result of the effects caused by atmospheric turbulence and the camera itself on the scene.

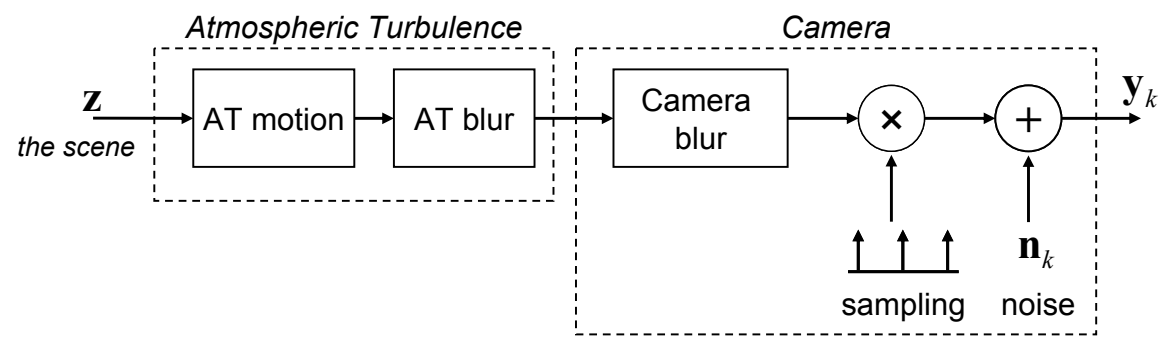

Figure 1. Flow diagram illustrating the degradation of a properly sampled scene $\mathbf{Z}$ into a turbulence and camera deteriorated image $\mathbf{y}_{k}$.

The degradation of camera frame $\mathbf{y}_{k}$ can be split up in two parts: a part that is due to the atmospheric turbulence and a part which is caused by the camera.

Atmospheric turbulence causes blurring, transformation (motion) and scintillation in the observed image of the scene. All these phenomena are assumed to be constant in a small region (patch) within the field-of-view. The size of such a region is determined by the isoplanatic angle, which is from a theoretical perspective, for a horizontal path through the atmosphere, often smaller than even a pixel. For the blurring component we simplified the model by assuming that it is only temporal varying, which is described by a Gaussian function with standard deviation $\sigma_{\mathrm{t}}(t)$. The transformation which is caused by turbulence is locally modeled by a translation. In this paper scintillation, the effect of illuminance variation, is not modeled.

The camera is modeled by a 'camera blur' (optics and sensor), a sampling device and additive Gaussian distributed noise. The camera blur is modeled by a Gaussian function with standard deviation $\sigma_{\mathrm{c}}$. In this paper it is assumed that the camera blur is small compared to the turbulence blur. Therefore the overall blur is assumed to be a Gaussian with standard deviation $\sigma_{\mathrm{tot}} \approx \sigma_{\mathrm{t}}$.

The imaging model as depicted in Figure 1 can be described by the following equation:

$$
\mathbf{y}_{k}=\mathbf{R} \mathbf{W}_{k} \mathbf{M}_{k} \mathbf{z}+\mathbf{n}_{k}
$$

where $\mathbf{M}_{k}$ represents the transformation matrix, $\mathbf{W}_{k}$ describes the blurring matrix and $\mathbf{R}$ is the resampling matrix, which is typically the identity matrix when no resolution enhancement takes place. 


\subsection{Proposed method}

This section describes the proposed turbulence compensation method. An overview of this method is depicted in Figure 2. The different steps are explained in more detail in the subsections below.

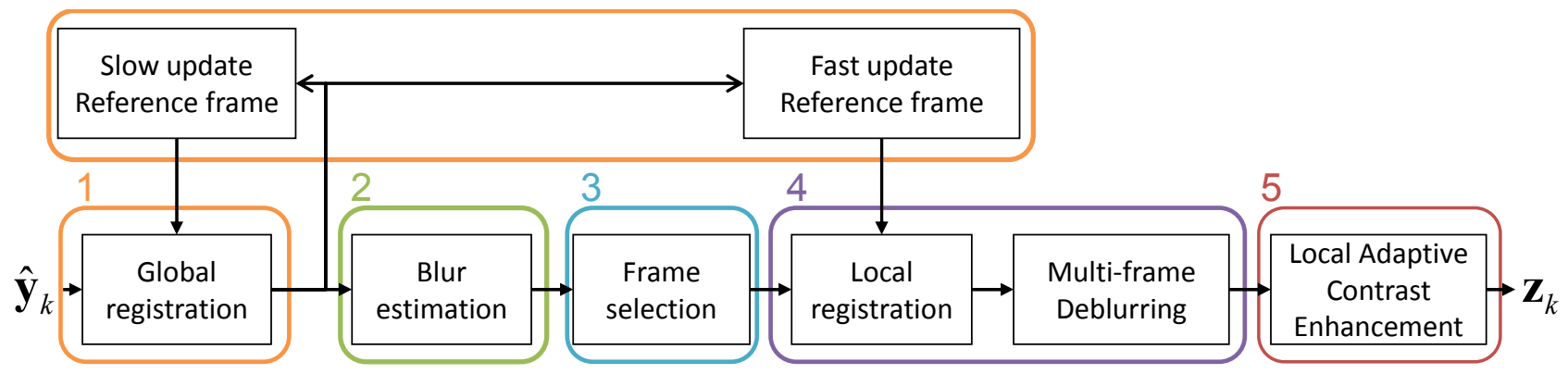

Figure 2. Flow diagram of the proposed method for compensation of the effects of atmospheric turbulence in images.

\subsubsection{Global registration and slow/fast update reference frame}

To stabilize the global structure of the scene a global registration [15] is performed assuming a translational motion model. This registration is done to a reference frame, which is slowly updated with registered input frames. With "slowly" we mean an update factor $\alpha$ in the order of 0.01 , according to:

$$
\mu_{i}=(1-\alpha) \mu_{i-1}+\alpha y_{s t a b}
$$

where $\mu_{i}$ is the reference frame at time $i$ and $y_{\text {stab }}$ is a stabilized input frame.

For performing local registration (in step 4) a fast updated reference frame is used. Here an update factor in the order of 0.1 is used. This fast update enables us to process slow moving objects in the scene. Both reference frames are initialized with the first frame of the sequence and are updated with registered input frames.

\subsubsection{Blur estimation}

In the second step the mean blur of each registered frame is estimated. This blur estimation is used later on (step 4) to perform the multi-frame deblurring. The method from Bouma et al. [16] is used which gives a precise and un-biased result.

\subsubsection{Frame selection}

The third step in the flow diagram of the proposed method is frame selection. This is done by calculating the $99^{\text {th }}$ percentile of the Gaussian gradient magnitude of each registered frame and selecting the $N_{s}$ out of $N_{t}$ frames with the highest percentiles. This results in an output sequence which is sharper and less fluctuating in sharpness compared to an output sequence without frame selection. Typical parameter setting ranges are $N_{s}=[10 . .50]$ and $N_{t}=[20 . .75]$ for frame rates between 30 and $70 \mathrm{~Hz}$.

\subsubsection{Local registration and Multi-frame Deblurring}

The fourth step consists local registration and multi-frame deblurring. Local registration is done using optical flow [17]. Optical flow estimates a per pixel local translation. To speed up computation of the optical flow first local translations are calculated at a sub-sampled and equally spaced grid in the image. From this grid the optical flow is interpolated. Typical parameter settings used are a grid space of 40 pixels and a window size of $64 \times 64$ pixels.

For multi-frame deblurring the method of Hardie et al. $[18,19]$ is used. The main difference is that our method uses optical flow registration while Hardie et al. uses global translation as registration. Equal to Hardie et al. the blurring is 
assumed spatially constant. For implementation reasons the mean blur of the selected $N_{s}$ frames is used in the optimization. To estimate the scene at frame $k\left(\mathbf{z}_{k}\right)$, the following cost function is minimized:

$$
C\left(\mathbf{z}_{k}\right)=\sum_{m}^{N_{s}}\left(\hat{\mathbf{y}}_{m}-\mathbf{R} \mathbf{W}_{k} \mathbf{M}_{k, m} \mathbf{z}_{k}\right)^{2}+\lambda_{r} \sum_{n}\left(L\left(\mathbf{z}_{k}\right)\right)^{2}
$$

where $\hat{\mathbf{y}}_{m}$ are $N_{s}$ observed frames used for deblurring, $\mathbf{M}_{k, m}$ represents the transformation matrix containing the global and local registration, $\mathbf{W}_{k}$ describes the blurring matrix and $\mathbf{R}$ is the resampling matrix. The final part of the cost function is the so-called regularization part which forces a smoothness constraint on the solution. Here $\lambda_{r}$ is a weighting factor and $L$ indicates a Laplacian operator. The cost function is minimized using a conjugate-gradient optimization. Typical parameter setting ranges for the deblurring part of our method are: resampling (upscaling) factor $=[1 . .4], \lambda_{r}=$ $\left[10^{-3} . .10^{-1}\right]$ and $[5 . .10]$ iterations for the conjugate-gradient minimization procedure.

\subsubsection{Local Adaptive Contrast Enhancement}

The last step in the flow diagram of the proposed method is local adaptive contrast enhancement (LACE) [20]. This method enhances the contrast locally based on the local variation in the scene.

\subsection{Computation time}

Note that the proposed method is implemented in MATLAB and is not optimized for speed. Processing time of estimating one turbulence compensated frame (one pipeline cycle) takes about 18 seconds on a Intel Xeon CPU E5620 @ $2.4 \mathrm{GHz}$ (64-bit) given a frame of $512 \times 512$ pixels. An optimized implementation is likely to run in real-time.

\section{QUANTITATIVE EVALUATION}

To evaluate the image quality improvement of the proposed turbulence compensation method the following measures are calculated: blur, tilt and Video Quality Measure (VQM). Because the available imagery does not contain special evaluation targets, such as TOD / MRTD charts, measures are selected that do not depend on such targets.

\subsection{Blur}

The mean blur in each frame is measured with the method described in [16]. For each frame the mean blur is reported as a Gaussian sigma in pixels (Gaussian blurring is assumed as indicated in Section 2.1). This mean blur is calculated in the original frames and in the processed frames as well. In this way an improvement / reduction of the blur can be calculated.

\subsection{Tilt}

The tilt is a measure indicating the amount of movement in the scene. This can be caused by camera movement, turbulence and moving objects in the scene. In the data we processed turbulence is the most prominent factor causing the tilt. The tilt is calculated with a gradient-based shift estimator [15] and reported as the length of the shift vector in pixels. Equal to the blur, the tilt is calculated for the original and processed images.

\subsection{Video Quality Measure}

The Video Quality Measure (VQM) is described in [13] and is specially designed for measuring improvement of image enhancement. Its main components are based on error estimates on edges and blur estimations. This measure is bounded by 0 (poor quality) and 1 (good quality). 


\section{RESULTS}

\subsection{Dataset}

The Dayton dataset is captured during a NATO SET-165 trial in Dayton, USA, beginning of October 2011 [21]. The distance over which the imagery is captured, is approximately $7 \mathrm{~km}$. An example of a measurement setup is depicted in Figure 3. The camera used is the monochrome AVT Stingray F-080B with a maximum frame size of $1032 \times 778$ pixels and a pixel depth of 14-bit. For the optics a Celestron C8 is used.

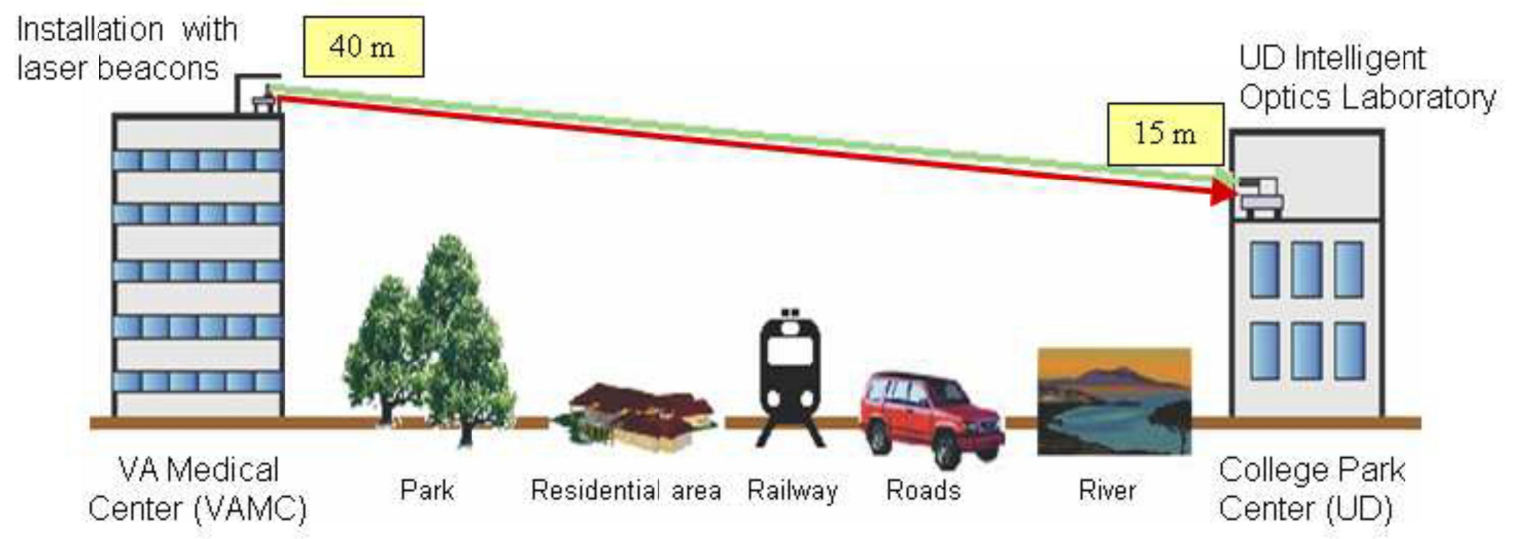

Figure 3. Graphical representation of the $7 \mathrm{~km}$ long atmospheric propagation path used for capturing some of the datasets.

From all the imagery captured during the trail, we selected and processed 10 datasets of different scenes under varying turbulence conditions. The datasets were captured on different days.

\subsection{Turbulence compensation}

To give an impression of the performance of the proposed turbulence compensation method, a few visual results are presented in the figures below. From these results it is clear that the imagery is much sharper after processing. The stabilization effect of the processing can only be appreciated by watching the processed sequence as a movie (see Figure $5)$.

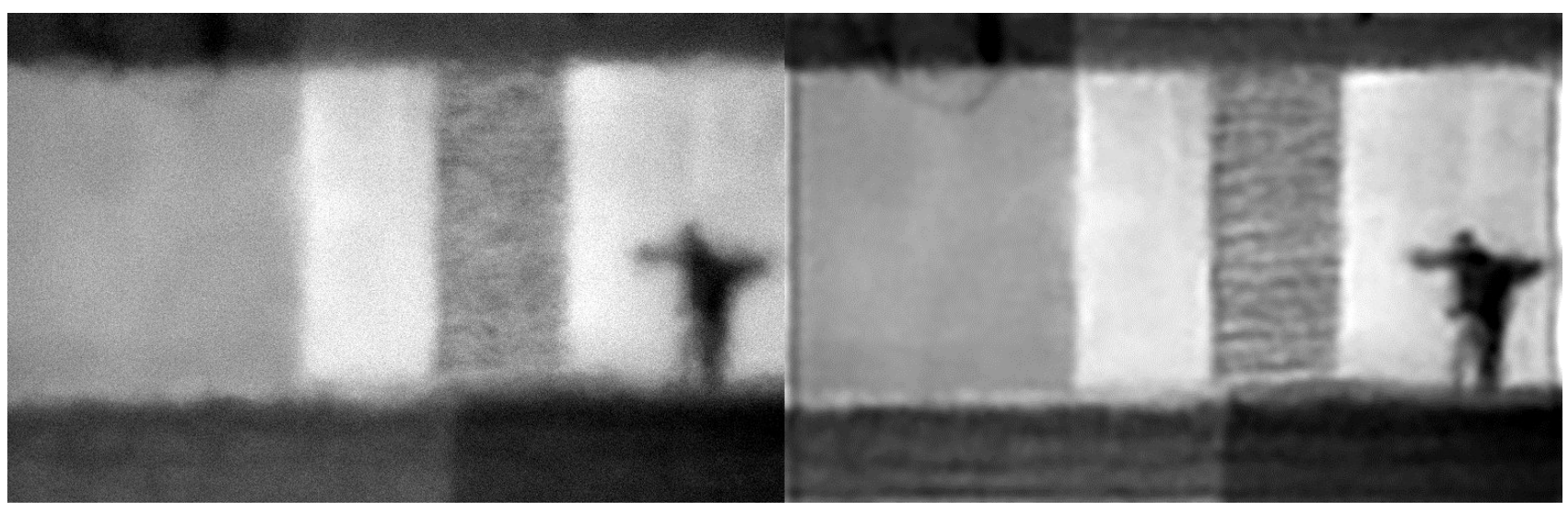

Figure 4. Left: one of the deteriorated frames of the "man-two-arms" sequence taken on 7 October 2011 at $11.19 \mathrm{~h}$ under heavy turbulence conditions. In this sequence the person is standing still. Right: corresponding frame processed by the proposed turbulence compensation method. 


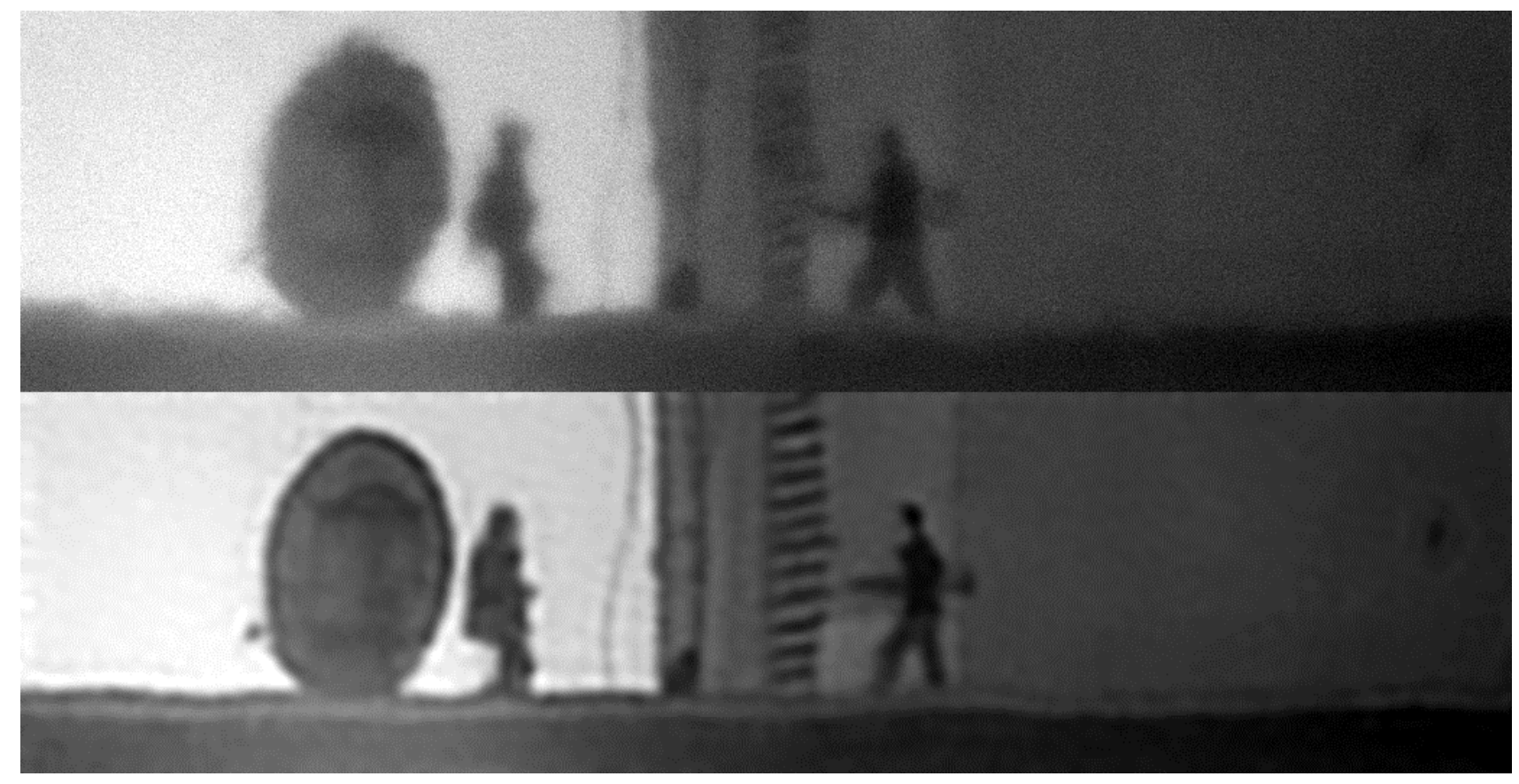

Figure 5. Top: one of the deteriorated frames of the "two-people-v1" sequence taken on 12 October 2011 at $10.56 \mathrm{~h}$ under medium turbulence conditions. In this sequence both persons are walking. Bottom: corresponding frame processed by the proposed turbulence compensation method. A video of this result is available at http://dx.doi.org/101117/12.2015371.1

\subsection{Evaluation}

After processing all 10 sequences, the improvement is analyzed using the measures described in Section 3. First an overview is given of the measured blur and tilt in the original and processed frames in Table 1.

The improvement (reduction) of the blur and tilt, together with the improvement of the VQM, is presented in Table 2.

Table 1. Measured mean blur and mean tilt in original and processed frames.

\begin{tabular}{|c|c|c|c|c|}
\hline Sequence name & $\begin{array}{l}\text { Blur org. ( } \sigma \\
\text { in pixels) }\end{array}$ & $\begin{array}{l}\text { Blur proc. ( } \sigma \\
\text { in pixels) }\end{array}$ & $\begin{array}{l}\text { Tilt org. } \\
\text { (pixels) }\end{array}$ & $\begin{array}{l}\text { Tilt proc. } \\
\text { (pixels) }\end{array}$ \\
\hline Man-two-arms & 6.5 & 6.2 & 2.9 & 0.2 \\
\hline Checkboard & 5.2 & 6.4 & 0.9 & 0.3 \\
\hline Two-people-v1 & 5.9 & 3.7 & 1.8 & 0.5 \\
\hline Two-people-v2 & 5.9 & 4.1 & 1.0 & 0.4 \\
\hline NATO & 4.4 & 3.4 & 3.1 & 0.3 \\
\hline Topantenna & 4.4 & 2.1 & 2.6 & 0.2 \\
\hline Stadium-lamps-200mm & 8.0 & 6.0 & 2.5 & 0.1 \\
\hline Stadium-lamps-66mm & 4.8 & 2.7 & 2.5 & 0.1 \\
\hline High-antenna-bottom & 4.1 & 3.4 & 1.3 & 0.3 \\
\hline High-antenna-top & 4.8 & 3.1 & 0.6 & 0.3 \\
\hline
\end{tabular}


Table 2. Overview of quality measures calculated on different sequences.

\begin{tabular}{|l|l|l|l|}
\hline Sequence name & Blur impr. & Tilt impr. & VQM impr. \\
\hline Man-two-arms & $1.0 \mathrm{x}$ & $16 \mathrm{x}$ & $0.4 \mathrm{x}$ \\
\hline Checkboard & $0.8 \mathrm{x}$ & $3.1 \mathrm{x}$ & $0.5 \mathrm{x}$ \\
\hline Two-people-v1 & $1.6 \mathrm{x}$ & $4.0 \mathrm{x}$ & $0.4 \mathrm{x}$ \\
\hline Two-people-v2 & $1.4 \mathrm{x}$ & $2.4 \mathrm{x}$ & $0.4 \mathrm{x}$ \\
\hline NAT0 & $1.3 \mathrm{x}$ & $9.3 \mathrm{x}$ & $0.6 \mathrm{x}$ \\
\hline Topantenna & $2.1 \mathrm{x}$ & $16 \mathrm{x}$ & $0.6 \mathrm{x}$ \\
\hline Stadium-lamps-200mm & $1.3 \mathrm{x}$ & $26 \mathrm{x}$ & $1.1 \mathrm{x}$ \\
\hline Stadium-lamps-66mm & $1.8 \mathrm{x}$ & $22 \mathrm{x}$ & $0.5 \mathrm{x}$ \\
\hline High-antenna-bottom & $1.2 \mathrm{x}$ & $4.1 \mathrm{x}$ & $0.5 \mathrm{x}$ \\
\hline High-antenna-top & $1.6 \mathrm{x}$ & $2.4 \mathrm{x}$ & $0.6 \mathrm{x}$ \\
\hline
\end{tabular}

From Table 2 it can be seen that the improvement in blur and tilt is significant due to the proposed turbulence compensation method. The blur is reduced between $0.8 \mathrm{x}-2.1 \mathrm{x}$ and shows no correlation with the amount of blur in the original scene. This indicates that the blur reduction is more or less constant with respect to the amount of blur. The tilt is reduced between $2.4 \mathrm{x}-26 \mathrm{x}$. These results correspond with the resulting imagery which looks much sharper and more stable (less shaky).

The improvement of the VQM $\left(=\mathrm{VQM}_{\text {proc }} / \mathrm{VQM}_{\text {org }}\right)$ indicates for almost every sequence a degradation of the image quality. This does not correspond with the visual results. Analyzing the VQM shows that the blur estimation part of this metric gives wrong estimates on our data. This makes this metric not suited for evaluating this type of data.

\section{CONCLUSIONS AND RECOMMENDATIONS}

In this paper a method is presented to compensate the effects of turbulence in image sequences. The results show qualitatively and quantitatively that the proposed method is capable of stabilizing and deblurring such image sequences. Analyzing 10 processed sequences show a blur reduction up to a factor of 2.1. Furthermore the scene is stabilized with a resulting tilt smaller than 0.5 pixel. These results enable an operator to see more details at large distances.

The proposed image quality evaluation methods for blur and tilt are simple and easy to use and give a good insight of the improvement of our method and correspond with visual perception. The VQM gives erroneous results on our data and is therefore not recommended.

\section{ACKNOWLEDGEMENTS}

The authors would like to thank the NATO SET-165 group for the availability of the Dayton dataset. 


\section{REFERENCES}

[1] Roggeman, M. C. and Welsh, B., "Imaging through turbulence," CRC Press, Boca Raton, USA, (1996).

[2] Eekeren, A. W. M. van, Schutte, K., Dijk, J., Schwering, P. B. W., Iersel, M. van, and Doelman, N. J., “Turbulence Compensation: an overview," Proc. SPIE Infrared Imaging Systems XXIII 8355, (2012) [doi:10.1117/12.918544].

[3] Schutte, K., Van Eekeren, A. W. M., Dijk, J., Schwering, P. B. W., Van Iersel, M., and Doelman, N. J., “An overview of turbulence compensation", (2012) [doi:10.1117/12.981942].

[4] Eekeren, A. W. M. van, Kruithof, M. C., Schutte, K., Dijk, J., Iersel, M. van, and Schwering, P. B. W., "Patchbased local Turbulence Compensation in anisoplanatic Conditions," Proc. SPIE Infrared Imaging Systems XXIII 8355, (2012) [doi:10.1117/12.918545].

[5] Eekeren, A. W. M. van, Schutte, K., and Schwering, P. B. W., "Turbulence Compensation enhances long-range imagery," SPIE Newsroom, (2012) [doi:10.1117/2.1201209.004481].

[6] Schwering, P. B. W., Broek, S. P. van den, and Iersel, M. van, "EO System Concepts in the Littoral," Proc. SPIE Infrared Technology and Applications XXXIII 6542, (2007).

[7] Iersel, M. van and Eijk, A. M. J. van, "Estimating turbulence in images," Proc. SPIE Free-Space Laser Communications X 7814, (2010).

[8] Huebner, C. S., "Compensating image degradation due to atmospheric turbulence in anisoplanatic conditions," Proc. SPIE Mobile Multimedia/Image Processing, Security, and Applications 7351, (2009).

[9] Zhu, X. and Milanfar, P., "Image Reconstruction from Videos Distorted by Atmospheric Turbulence," Proc. SPIE Visual Information Processing and Communication 7543, (2010).

[10] Bijl, P., Schutte, K., and Hogervorst, M. A., "Applicability of TOD, MTDP, MRT and DMRT for dynamic image enhancement techniques," in Infrared Imaging Systems: Design, Analysis, Modeling and Testing XVII 6207, (2006).

[11] Krumpholz, M. and Katehi, L. P. B., "MRTD: new time-domain schemes based on multiresolution analysis," Microwave Theory and Techniques, IEEE Transactions on 44(4), 555 -571, (1996) [doi:10.1109/22.491023].

[12] Wang, Z., Bovik, A. C., Sheikh, H. R., and Simoncelli, E. P., "Image quality assessment: from error visibility to structural similarity," Image Processing, IEEE Transactions on 13(4), 600 -612, (2004) [doi:10.1109/TIP.2003.819861].

[13] Baumgartner, D. D. and Schachter, B. J., "Improving FLIR ATR performance in a turbulent atmosphere with a moving platform," Proc. SPIE 8391, (2012) [doi:10.1117/12.917197].

[14] Dijk, J., Schutte, K., Van Eekeren, A. W. M., and Bijl, P., "Measuring the performance of super-resolution reconstruction algorithms," in Society of Photo-Optical Instrumentation Engineers (SPIE) Conference Series 8355, p. 36, (2012).

[15] Pham, T. Q., Bezuijen, M., Vliet, L. J. van, Schutte, K., and Hendriks, C. L. L., "Performance of optimal registration estimators," Proc. SPIE Visual Information Processing XIV 5817, 133-144, (2005).

[16] Bouma, H., Dijk, J., and Eekeren, A. W. M. van, "Precise local blur estimation based on the first-order derivative," Proc. SPIE Visual Information Processing XXI 8399, (2012).

[17] Horn, B. K. P. and Schunck, B. G., "Determining optical flow," Artificial Intelligence 17, 185-203, (1981).

[18] Hardie, R. C., Barnard, K. J., Bognar, J. G., Armstrong, E. E., and Watson, E. A., "High-resolution image reconstruction from a sequence of rotated and translated frames and its application to an infrared imaging system," Optical Engineering 37(1), 247-260, (1998).

[19] Eekeren, A. W. M., Schutte, K., Oudegeest, O. R., and Vliet, L. J. van, "Performance evaluation of SuperResolution reconstruction methods on real-world data," EURASIP Journal on Advances in Signal Processing, 111, (2007).

[20] Schutte, K., "Multiscale adaptive gain control of IR images," Proc. SPIE Infrared Technology and Applications XXIII 3061, (1997).

[21] Velluet, M. T., Vorontsov, M., Espinola, R., Marchi, G., Nicolas, S., Schwering, P., and Riker, J., "Turbulence characterization and image processing data sets from a NATO RTO SET 165 trial in Dayton, OH, USA," Proc. SPIE Atmospheric Propagation IX 8380, (2012). 